\title{
NORMED LINEAR SPACES EQUIVALENT TO INNER PRODUCT SPACES
}

\section{J. T. JOICHI ${ }^{1}$}

1. Introduction. There are many conditions which are known to characterize those normed linear spaces (NLS) $X$ which are inner product spaces (IPS), that is, conditions under which it is possible to define an inner product in $X$ in such a way that it will induce the given norm (cf. [1]). However, little is known about when a NLS $X$ is merely equivalent to an IPS, that is, when it is possible to define an inner product in $X$ in such a way that the induced norm is equivalent to the given norm. Two norms $|\cdot|_{1}$ and $|\cdot|_{2}$ in $X$ are called equivalent if there exists a constant $k \geqq 1$ so that $(1 / k)|x|_{1} \leqq|x|_{2}$ $\leqq k|x|_{1}$ for each $x$ in $X$. The only such characterizations known to us are those in [3], [4], and [5]. In this paper, we give another such characterization. The statement and proof of our main theorem are in $\S 3$. In $\$ 2$ we prove a preliminary result concerning the existence of invariant means on a certain space of bounded real-valued functions.

2. Invariant means. Let $(S, \geqq)$ be a semilattice, that is, a partially ordered set (reflexive, antisymmetric and transitive) in which every pair of elements have a least upper bound (write: $s \vee t=1$.u.b. $\{s, t\}$ ). Let $m(S)$ be the Banach space of bounded real-valued functions defined on $S$ with the supremum norm. Since $S$ is a directed set, each element $f$ in $m(S)$ is a net and we let $c(S)$ be the closed linear subspace of $m(S)$ consisting of those functions which are convergent nets.

For $f$ in $c(S)$, let $\phi(f)=\lim _{S} f$. Then $\phi$ is a bounded linear functional on $c(S)$. For $f$ in $m(S)$, let

$$
\begin{aligned}
& p(f)=\lim \sup _{s} f \equiv \lim _{S}[\sup \{f(t): t \geqq s\}] \\
& q(f)=\lim \inf _{S} f \equiv \lim _{S}[\inf \{f(t): t \geqq s\}] .
\end{aligned}
$$

For each $s$ in $S$, let $L$, be the linear operator in $m(S)$ defined by,

$$
\left(L_{s} f\right)(t)=f(s \vee t) \text {. }
$$

It is easily seen that,

(i) $\left\{L_{s}: S\right.$ in $\left.S\right\}$ is a commutative semigroup of bounded linear operators on $m(S)$,

(ii) $L_{s}(c(S)) \subseteq c(S)$, for each $s$ in $S$,

Received by the editors July 19, 1965.

1 This research was supported in part by the National Science Foundation under grant G24295. 
(iii) $\phi\left(L_{s} f\right)=\phi(f)$, for each $s$ in $S$ and $f$ in $c(S)$, and

(iv) $p\left(L_{s} f\right)=p(f)$, for each $s$ in $S$ and $f$ in $m(S)$.

Since, in addition, $\phi(f)=p(f)$ for each $f$ in $c(S)$, by a generalization of the Hahn-Banach Theorem due to M. A. Woodbury [7] (cf. [6, p. 164]), there exists an extension $\Phi$ of $\phi$ to $m(S)\left(\Phi\right.$ in $\left.m(S)^{*}\right)$ such that

(a) $\Phi(f) \leqq p(f)$ for each $f$ in $m(S)$, and

(b) $\Phi\left(L_{s} f\right)=\Phi(f)$ for each $s$ in $S$ and $f$ in $m(S)$.

From (a) it follows that $q(f)=-p(-f) \leqq \Phi(f)$, that is,

(c) $q(f) \leqq \Phi(f) \leqq p(f)$ for each $f$ in $m(S)$.

\section{The main theorem.}

Theorem. Let $X$ be a NLS. Then a necessary and sufficient condition that $X$ be equivalent to an IPS is that there exist a constant $k \geqq 1$ such that for each finite dimensional subspace $M$ of $X$, there exists a linear mapping $T_{M}$ of $M$ into $H$ (Hilbert space) such that $(1 / k)|x|$ $\leqq\left|T_{M} x\right| \leqq k|x|$ for each $x$ in $M$.

Proof. The necessity is obvious. To prove the sufficiency, let $S$ be the set of finite dimensional subspaces of $X$ ordered by containment. Then $S$ is a semilattice where $M \bigvee N=M+N$ (vector sum) for $M$ and $N$ in $S$. Thus, by the results of the previous section, there exists a functional $\Phi$ in $m(S) *$ satisfying conditions (a), (b) and (c).

For $x$ in $X$, define $f_{x}$ in $m(S)$ by,

$$
f_{x}(M)=\left\{\begin{array}{cl}
\left|T_{M} x\right| & \text { if } x \text { is in } M, \\
0 & \text { if } x \text { is not in } M .
\end{array}\right.
$$

Now, let $n(x)=\left[\Phi\left(f_{x}^{2}\right)\right]^{1 / 2}$. We wish to show that $n$ is an inner product norm in $X$ equivalent to the original one.

Clearly, $n$ is a nonnegative function and $n(\alpha x)=|\alpha| n(x)$ for any vector $x$ and scalar $\alpha$. Also, for any vector $x$, we have

$$
n(x)^{2}=\Phi\left(f_{x}^{2}\right)\left\{\begin{array}{l}
\leqq p\left(f_{x}^{2}\right)=\left[\lim \sup _{S} f_{x}\right]^{2} \leqq[k|x|]^{2} \\
\geqq q\left(f_{x}^{2}\right)=\left[\lim \inf _{S} f_{x}\right]^{2} \geqq[(1 / k)|x|]^{2},
\end{array}\right.
$$

so,

$$
(1 / k)|x| \leqq n(x) \leqq k|x| \text {. }
$$

Thus, $n(x)=0$ if and only if $x=0$, and if $n$ is a norm, it is equivalent to the original one. To show that $n$ is a norm, it remains to show that $n$ satisfies the triangle law. This will follow once we show that $n$ satisfies the parallelogram law (and hence, is an inner product norm). 
For vectors $x$ and $y$ in $X$, let $N$ be the subspace spanned by $x$ and $y$. Then, for any subspace $M$ such that $M \supseteq N$, we have

$$
\begin{aligned}
2\left[f_{x}^{2}(M)+f_{y}^{2}(M)\right] & =2\left[\left|T_{M} x\right|^{2}+\left|T_{M} y\right|^{2}\right] \\
& =\left|T_{M} x-T_{M} y\right|^{2}+\left|T_{M} x+T_{M} y\right|^{2} \\
& =f_{x-y}^{2}(M)+f_{x+y}^{2}(M)
\end{aligned}
$$

where the second equality follows since $T_{M}(M)$ is a subspace of Hilbert space and the norm in a Hilbert space does satisfy the parallelogram law. Applying $\Phi$, it follows that

$$
2\left[n(x)^{2}+n(y)^{2}\right]=n(x-y)^{2}+n(x+y)^{2}
$$

that is, $n$ satisfies the parallelogram law.

Now, let $B=\{x: n(x) \leqq 1\}$. Then $n$ is the Minkowski functional of $B$ and will satisfy the triangle law if $B$ is convex (cf. [1, p. 11]). To see that $B$ is convex, we note first that although the mapping $x \rightarrow f_{x}$ of $X$ into $m(S)$ is not continuous, the mapping $x \rightarrow n(x)$ of $X$ into the real numbers is continuous. Suppose now that $x$ and $y$ are vectors with $n(x)=n(y)=1$ and for some $\alpha$ with $0<\alpha<1$, we have $n(\alpha x+(1-\alpha) y)>1$. Then, by the continuity of $n$, there exists $\alpha_{1}$ and $\alpha_{2}$ so that $0 \leqq \alpha_{1}<\alpha<\alpha_{2} \leqq 1, n\left(\alpha_{i} x+\left(1-\alpha_{i}\right) y\right)=1$ for $i=1,2$, and if $\alpha_{1}<\beta<\alpha_{2}$ then $n(\beta x+(1-\beta) y)>1$. Let $x_{i}=\alpha_{i} x+\left(1-\alpha_{i}\right) y$ for $i=1,2$. Then, by the parallelogram law, we have

$$
\begin{aligned}
4 & =2\left[n\left(x_{1}\right)^{2}+n\left(x_{2}\right)^{2}\right]=n\left(x_{1}+x_{2}\right)^{2}+n\left(x_{1}-x_{2}\right)^{2} \\
& \geqq n\left(x_{1}+x_{2}\right)^{2}=4 n\left((1 / 2)\left(x_{1}+x_{2}\right)\right)^{2}>4 .
\end{aligned}
$$

This contradiction establishes the convexity of $B$.

Corollary. Let $X$ be a NLS. Then a necessary and sufficient condition that $X$ be equivalent to an IPS is that there exists a constant $k \geqq 1$ such that for each pair of finite dimensional subspaces $M$ and $N$ with $\operatorname{dim} M=\operatorname{dim} N$, there exists a linear mapping $T$ of $M$ onto $N$ such that $(1 / k)|x| \leqq|T x| \leqq k|x|$ for each $x$ in $M$.

This corollary is an immediate consequence of our theorem and the following theorem due to Dvoretzky [2].

Theorem (Dvoretzky). Given $\epsilon(0<\epsilon<1)$ and a positive integer $m$, there exists an integer $N=N(m, \epsilon)$ such that if $C$ is any symmetric convex body in $E^{n}$ (real Euclidean $n$-space), where $n \geqq N$, then there is a subspace $E^{m}$ and a positive number $r$ so that $B_{r(1-c)} \subseteq C \cap E^{m} \subseteq B_{r}$, where $B_{8}=\left\{x\right.$ in $\left.E^{m}:|x| \leqq s\right\}$. 
REMARK. We note that our theorem is applicable to any NLS whether or not it be separable. Also, the mappings $T_{M}$ need only exist for some semilattice $S$ of finite dimensional subspaces $M$ of $X$ such that the vector subspace $U\{M: M$ in $S\}$ is dense in $X$. In case $X$ is separable, the mappings $T_{M}$ need only exist for a sequence $\left\{M_{n}\right\}$ of finite dimensional subspaces such that $M_{n+1} \supseteq M_{n}$ and $\cup\left\{M_{n}: n=1,2, \cdots\right\}$ is dense in $X$. In this latter case, the functional $\Phi$ is an ordinary Banach limit on the Banach space of bounded sequences.

\section{REFERENCES}

1. M. M. Day, Normed linear spaces, Springer, Berlin, 1958.

2. A. Dvoretzky, $A$ theorem on convex bodies and applications to Banach spaces, Proc. Nat. Acad. Sci. U.S.A. 45 (1959), 223-226.

3. Jesús Gil de Lamadrid, On finite dimensional approximations of mappings in Banach spaces, Proc. Amer. Math. Soc. 13 (1962), 163-168.

4. S. Kakutani and G. W. Mackey, Ring and lattice characterizations of complex Hilbert space, Bull. Amer. Math. Soc. 52 (1946), 727-733.

5. J. Lindenstrauss, On the modulus of smoothness and divergent series in Banach spaces, Michigan Math. J. 10 (1963), 241-252.

6. H. L. Royden, Real analysis, Macmillan, New York, 1963.

7. M. A. Woodbury, Invariant functionals and measures, Abstract 168T, Bull. Amer. Math. Soc. 56 (1950), 172.

University of Minnesota 